\title{
Poloidal correlation reflectometry at W7-X: Radial electric field and coherent fluctuations
}

\author{
T Windisch ${ }^{1}$, A Krämer-Flecken ${ }^{2}$, JL Velasco ${ }^{3}$, A Könies ${ }^{1}$, \\ C Nührenberg ${ }^{1}$, O Grulke ${ }^{1}$, T Klinger ${ }^{1}$, and the $W^{\top}$ - $X$ team \\ ${ }^{1}$ Max-Planck-Institute for Plasma Physics, D-17491 Greifswald, Germany \\ ${ }^{2}$ Forschungszentrum Jülich, D-52428 Jülich, Germany and \\ ${ }^{3}$ Laboratorio Nacional de Fusin, CIEMAT, E-28040 Madrid, Spain
}

(Dated: May 19, 2017)

\begin{abstract}
Poloidal correlation reflectometry measurements during the first plasma campaign of the optimized stellarator Wendelstein-7X are presented. The radial electric field is determined and a comparison with neoclassical calculations shows good qualitative agreement. The measured density fluctuation spectrum exhibits coherent low- and high-frequency modes. Magneto-hydrodynamic (MHD) modeling results suggest that the coherent fluctuations are caused by stable MHD-modes and Alfvén waves, respectively.

PACS numbers: 52.35.Kt,52.35.Ra,52.25.Xz
\end{abstract}

*Electronic address: thomas.windisch@ipp.mpg.de 


\section{INTRODUCTION}

While in the past decades axis-symmetric tokamak devices were in the focus of magnetic fusion research stellarators start to catch up. To a large extent, this development is caused by successful neoclassical optimization of transport losses due to plasma particles trapped in the helical ripple of the non-axisymmetric magnetic field topology of stellarators $[1,2]$. Stellarator optimization became a broad research field [5], and Wendelstein 7-X (W7-X) has almost a quasi-isodynamic equilibrium. Here the bounce-averaged radial drift of trapped particles is greatly reduced while the current component parallel to the magnetic field remains small, which allows a stiff equilibrium at higher plasma pressures [3]. In stellarators the radial electric field $E_{r}$ is a key quantity for the neoclassical transport. It is determined by the ambipolarity constraint of neoclassical fluxes. In contrast to axi-symmetric devices, particle fluxes in helical devices strongly depend on Er and this sets a constraint on the radial electric field for given plasma density and temperature profiles, which has multiple possible solutions [13]. In the low-collisionality long-mean-free-path (lmfp) regime, a solution with positive $E_{r}$ in the plasma core (with $T_{e} \gg T_{i}$ ) can be accessed by strong electron heating with electron cyclotron resonance heating $(\mathrm{ECRH})$. In the plasma edge (with $T_{e} \approx T_{i}$ ) the relatively enhanced ion flux requires a negative $E_{r}$ in order to maintain ambipolarity. For the first operation phase W7-X was equipped with five inboard graphite limiters (at minor radius $a=0.49 \mathrm{~cm}$ ) to protect other in-vessel components [14]. This has imposed a limit to the total absorbed plasma heating energy to $4 \mathrm{MW}$ (ECRH only with $P_{E C R H} \leq 4.3 \mathrm{MW}$ ) and at the resulting low plasma densities both solutions for $E_{r}$ are simultaneously observed. While neoclassical transport typically dominates the plasma core, the plasma edge can be governed by turbulent transport as caused by microinstabilities that are driven by radial pressure gradients. In W7-X configuration some microinstabilities are predicted to be more stable if compared to a s tokamak device of the same size [6].

Poloidal Correlation Reflectometry (PCR) is well suited to characterize the afore mentioned aspects. It allows one to measure the propagation speed of density fluctuations from which, under certain assumptions, the radial electric field can be derived. It also yields important fluctuation characteristics, such as frequency and wavenumber spectra as well as correlation lengths and times, that can be utilized to investigate the underlying instability mechanisms and compared with theoretical predictions. The structure of the paper aims 
to reflect both aspects also to highlight the capability of the PCR system. In Sec. II the experimental setup and data evaluation of the PCR system is described. In Sec. III experimental results with focus on radial electric field $E_{r}$ and coherent low- and high-frequency fluctuations are presented. The measured $E_{r}$ is compared to neoclassical predictions in the monoenergetic limit using the DKES code [7]. First experimental results have been recently published also elsewhere [32]. In this paper the origin of the observed coherent fluctuations is investigated in detail using Mirnov coil data [21] and compared to MHD code results using CAS3D [4] and CONTI [27]. The paper is summarized in Sec. IV.

\section{EXPERIMENTAL SETUP AND DATA ANALYSIS}

\section{A. Microwave generation and detection}

Poloidal correlation reflectometry is a non-invasive microwave diagnostic that utilizes a poloidal array of microwave antennae to investigate the spatio-temporal characteristics of plasma density fluctuations [15]. The system operates in the $K$ and $K_{a}$-band $\left(f_{c}=22-\right.$ $40 \mathrm{GHz})$ using ordinary wave polarization $(O$-mode) which translates into cut-off densities $n_{c o}=\epsilon_{0} m_{e}(2 \pi f)^{2} / e^{2}=0.7-2 \cdot 10^{19} \mathrm{~m}^{-3}$. A schematic diagram of the microwave generator is displayed in Fig. 1. It consists of two microwave sources (voltage controlled oscillators, VCO) covering the range $10-20 \mathrm{GHz}$ which are phase coupled by a phase-locked-loop (PLL). After one stage of frequency multiplication, amplification and band-pass filtering, the output power is typically $P_{\text {out }} \approx 100 \mathrm{~mW}$. For the heterodyne detection both sources $f_{1,2}$ are slightly detuned to produce an intermediate beat frequency (IF) of $f_{1}-f_{2}=60 \mathrm{MHz}$ after frequency multiplication. The IF and the $60 \mathrm{MHz}$ reference signal from the generator finally feed the quadrature detector. The output of the quadrature detector $(I Q$-signal) is digitized with a sampling frequency of $4 \mathrm{MHz}$ and 14bit resolution. Data acquisition and control of the microwave synthesizer is done via MDSplus. The frequency of the microwave generator is either constant or hopped between various frequency plateaus. In the latter operation mode the hopping time is typically set to $t_{\text {hop }}=5-20 \mathrm{~ms}$. The switching time between two arbitrary frequencies is less than $0.1 \mathrm{~ms}$. 


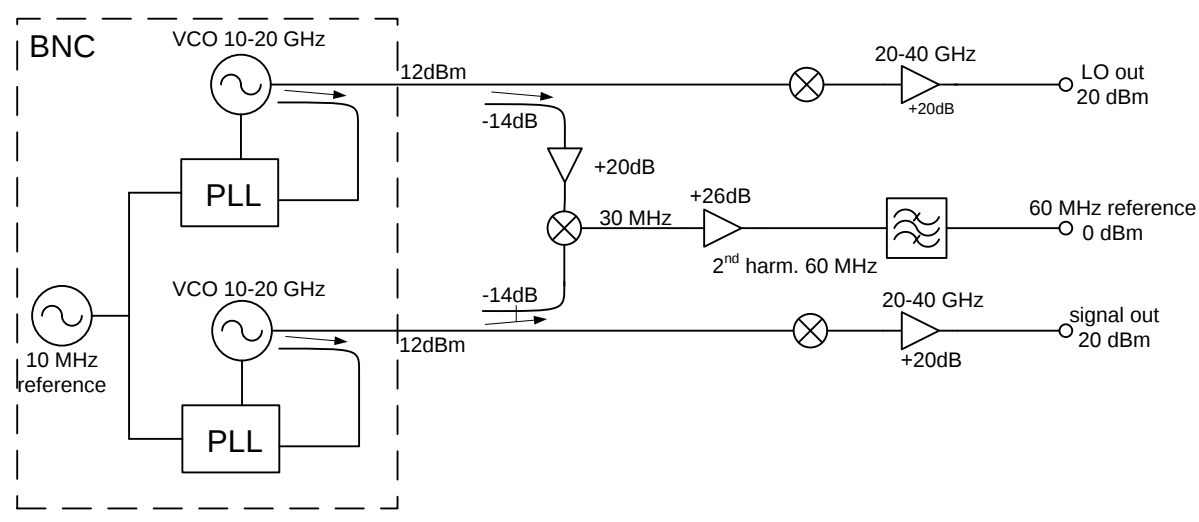

FIG. 1: Schematic setup of microwave generation.

\section{B. Antenna array}

The antenna array consists of five pyramidal horn antennae (mouth dimensions $44.1 \times 34.8 \mathrm{~mm})$. The schematic design of the antenna array and the support structure is illustrated in Fig. 2a. Antenna A denotes the launching antenna which is connected with the signal output of the microwave generator. Antennae B-E denote the receiving antennae which measure the microwave radiation reflected from the cut-off layer.

The antenna array is localized in the elongated bean-shaped plane $\left(\varphi=72^{\circ}\right)$, in which the magnetic field approximately scales as $1 / R$ (with $R$ denoting major radius). The orientation of the antennae line-of-sights is indicated in Fig. 2b. The line-of-sight of antenna A is designed to be aligned normal to the flux surface of the vacuum magnetic field configuration at the design cut-off layer $r / a \approx 0.95-1$ ( $a=0.49 \mathrm{~m}$ denotes the minor radius). Note that $r=a \sqrt{\Psi / \Psi_{a}}$, where $\Psi$ is the toroidal flux and $a, \psi$ and $\psi_{a}$ are provided by VMEC [7].

In the limiter magnetic configuration used during the first plasma campaign, the magnetic pitch angle at the design cutoff layer is $\alpha=\tan ^{-1}\left(B_{z} / B_{\varphi}\right) \approx 13^{\circ}$. The design orientation of the other antennae is such that they have a joint focus with the line-of-sight of antenna A at $r / a \approx 0.32$ (cf. Fig. 2b). The viewing geometry of all antennae has been verified under laboratory conditions. In the far field the emission pattern can approximated by a Gaussian beam with beam waist $w_{0}=1.65 \mathrm{~cm}$ at $f_{c}=30 \mathrm{GHz}$. 


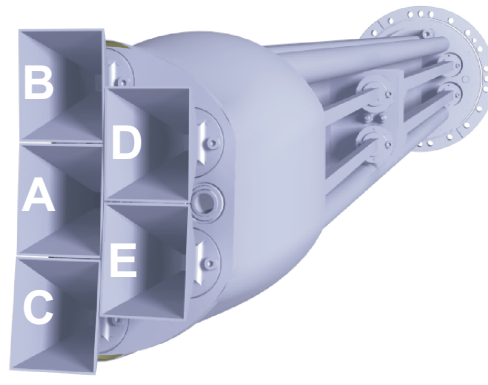

a)

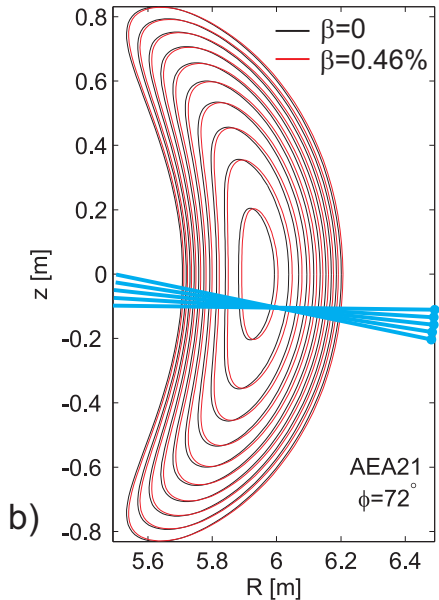

FIG. 2: (a) CAD illustration of the PCR plugin including the antenna nomenclature. (b) Fluxsurfaces of the OP1.1 configuration in the bean plane at $\varphi=72^{\circ}$ for two values of the plasma- $\beta$ $(<\beta>=0$ and $<\beta>=0.46 \%)$. The antennae array and lines-of-sight are indicated in blue.

\section{Radial localization}

The radial localization of the cutoff layer is determined by the radial plasma density profile which is measured by a Thomson scattering system [17]. Typical profiles of electron density and temperature are shown in Fig. $3 \mathrm{c}$ and $\mathrm{d}$ for three time instants of a plasma discharge (program id 160303.013). The corresponding time traces of ECRH power $P_{E C R H}$ and line integrated density $\int n d l$ are shown in Fig. 3a and b, respectively. After a transient heating phase in the beginning of the discharge, the ECRH power remains constant at
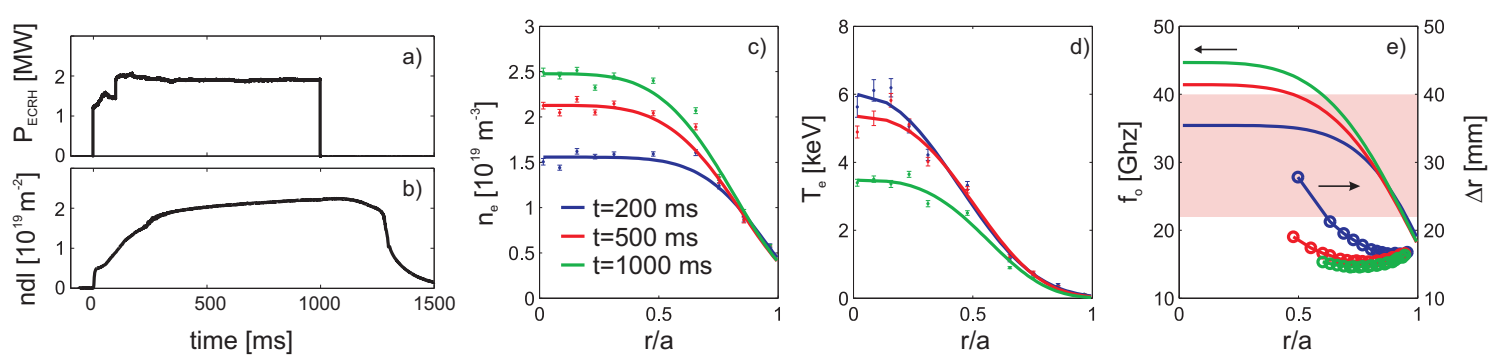

FIG. 3: Time traces of ECRH-heating power (a) and line integrated density (b) for a plasma discharge (program id 160303.013). Radial profiles of $n_{e}(\mathrm{c})$ and $T_{e}(\mathrm{~d})$ at $t=200,500,1000 \mathrm{~ms}$. The cut-off frequencies are shown in e) together with the radial resolution $\Delta r$. The $K_{a}$-band is indicated by the shaded box. 
$P_{E C R H}=2 \mathrm{MW}$. Throughout the discharge the line-integrated density slowly rises due to wall recycling and outgasing caused by incomplete wall conditioning during the first operation campaign. The peak electron density increases from $n_{e} \approx 1.5 \cdot 10^{19} \mathrm{~m}^{-3}$ at $t=$ $200 \mathrm{~ms}$ to $n_{e} \approx 2.5 \cdot 10^{19} \mathrm{~m}^{-3}$ at $t=1000 \mathrm{~ms}$ while the central electron temperature drops from $6 \mathrm{keV}$ to $3.5 \mathrm{keV}$ due to enhanced coupling to the ions at higher Coulomb collisionality. The corresponding $O$-mode cut-off frequencies $2 \pi f_{c}=\sqrt{e^{2} n_{e} /\left(\epsilon_{0} m_{e}\right)}$ and the radial resolution are depicted in Fig. 3e for the three time instants. For the given profiles, the radial region accessible in the utilized microwave band is $r / a \approx 0.5-1$. The radial resolution is given by $\Delta r=L_{n} /\left((\omega / c) L_{n}\right)^{2 / 3}$, where $L_{n}$ is the radial density gradient length [19]. Due to the shallow density profile, $\Delta r$ is rather poor $(\Delta r \approx 15-30 \mathrm{~mm})$.

\section{Data analysis}

The data analysis method comprises two main elements, i.e., the determination of the reflection point position on the flux surface for various antenna combinations and the derivation of the corresponding time lag $\tau$. In order to calculate the velocity, knowledge of the precise measurement location and its orientation relative to the local magnetic field is crucial. Antenna A launches a rather broad Gaussian beam that is reflected at the cut-off layer towards the receiving antennae B,C,D and E. The reflection points are calculated using the 3D ray tracing code TRAVIS [18], which uses as input the $n_{e^{-}}$and $T_{e}$-profiles, the
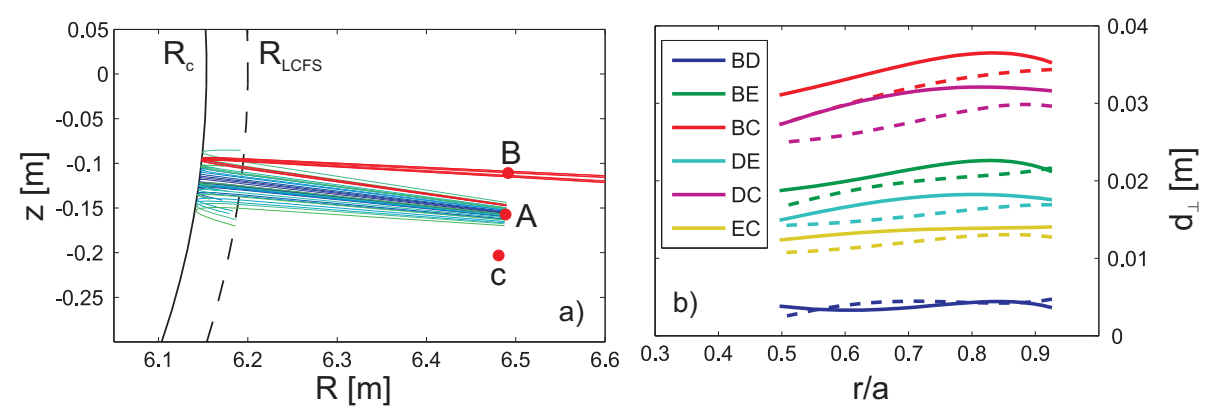

FIG. 4: a) TRAVIS ray tracing paths for $f_{c}=28 \mathrm{GHz}$ in the poloidal plane (program id 160303.013 at $t=500 \mathrm{~ms}$ ). The positions of antennae A,B and $\mathrm{C}$ are shown as labeled red dots. The black line indicates the cut-off position at $r / a=0.83$ and the dashed black line indicates the last closed flux surface (LCFS). b) Radial profile of estimated $d r_{\perp}$ for all antenna combinations using TRAVIS (solid) and the simplified geometric approach (dashed). 

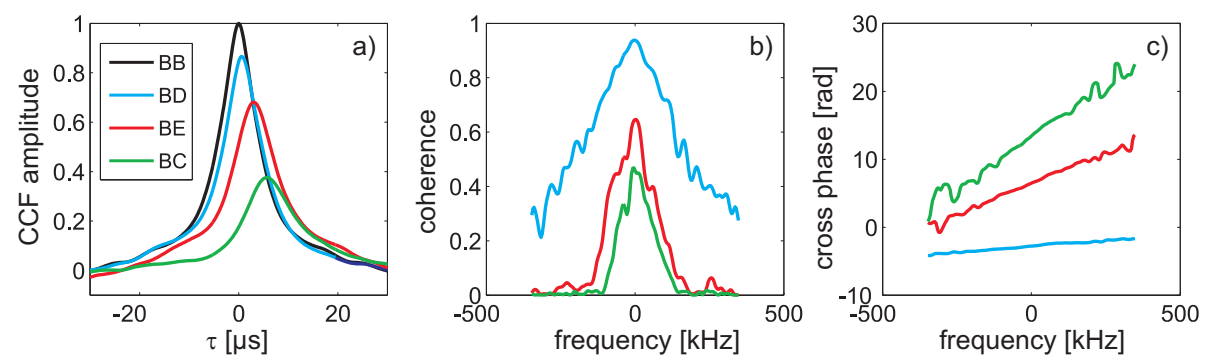

FIG. 5: (a) Normalized cross-correlation function (CCF), (b) cross-coherence and (c) cross-phase spectra for antenna B (program id 160303.013, $t=600-610 \mathrm{~ms}$ )

magnetic configuration, the antenna geometry, and the microwave frequency $f_{c}$. Despite the limitations of the WKB approximation in the radial region close to the cut-off it can be demonstrated that the TRAVIS ray directions far away from the cut-off, i.e. in the measurement plane, are in good agreement with full-wave simulations (not shown here), which further allow to investigate the wave structure modification in this region. For all results presented in this paper, the vacuum magnetic field is used since plasma- $\beta$ effects are rather small in the measurement region (typically $\langle\beta\rangle=0.3 \%$, cf. Fig. 2b). TRAVIS is run in beam trace mode in which the Gaussian beam propagation is approximated by several weighted individual rays, aligned cylindrically around the central ray at the launching position. A sample TRAVIS calculation is shown in Fig. 4a for a microwave frequency $f_{c}=28 \mathrm{GHz}$ (program id 160303.013 at $\left.t=500 \mathrm{~ms}\right)$. The Gaussian beam $\left(w_{0}=1.65 \mathrm{~cm}\right)$ is launched from antenna $\mathrm{A}$, is refracted in the plasma and reflected at the cut-off layer towards the receiving antennae. The rays are traced until they eventually intersect with one of the receiving antenna mouths. For each antenna the reflection point on the flux surface is calculated using a weighting of all intersecting rays according to their incident power. To ensure that enough reflected rays intersect with all receiving antenna mouths a large number (typically $N=1296$ ) single rays contribute to the Gaussian beam. In Fig. 4a a subset of all launched rays is shown in green, while the rays used to obtain the reflection point of antenna B are indicated in red. The various reflection points are then mapped to the local magnetic field in order to obtain the perpendicular distance $d_{\perp}$ used to calculate $v_{\perp}=d_{\perp} / \tau$. By changing $f_{c}$ the entire accessible density profile is covered. The resulting $d_{\perp}$-profile for all six possible antenna combinations is shown in Fig. 4b as solid lines. Also shown is an evaluation using a simplified approach using geometrical optics neglecting refraction. The 
differences between the two methods depends strongly on the density profile but can be significant for the derivation of $v_{\perp}$.

The measurement of the time lag $\tau$ relies on the cross-correlation function (CCF) technique. In order to remove artefacts that would occur in the frequency hopping mode, the data is band-pass filtered in the range $f_{c}=[1,350] \mathrm{kHz}$. The CCF amplitudes for antenna $\mathrm{B}$ are shown in Fig. 5a (program id 160303.013 at $t=600-610 \mathrm{~ms}$ ). With increasing distance between the reflection points, the CCF amplitude decreases and the maximum develops a positive time lag. From the decrease of the CCF amplitude a poloidal correlation length $l_{c}=4.5 \mathrm{~cm}$ is obtained. If the density fluctuation spectrum has some distinct features, e.g., high frequency components with small magnitude, the CCF analysis might not be the right choice to characterize the phase relation between the antenna combinations at this frequency. For this purpose the coherence $C$ and cross-phase $\phi$ can be used, which are shown in Fig. 5b and Fig. 5c, respectively. The data is the same as for the CCF analysis. For antenna combination $\mathrm{BD}$ the distance between the reflection points is smallest which causes a high coherence over the whole frequency range. For the other combinations BE and $\mathrm{BC}$ the highest coherence value decreases to 0.6 and 0.4 , respectively, as expected from the CCF analysis. From the linear slope of the cross-phase, the time lag is obtained via the group delay $2 \pi \tau_{\phi}=d \phi / d f$. The agreement between the different approaches is very good, for example $\tau_{C C F}=3.0 \mu \mathrm{s}$ and $\tau_{\phi}=2.9 \mu \mathrm{s}$ for antenna combination BE.

\section{RESULTS}

\section{A. Fixed frequency operation}

As described Sec. II A the PCR system can be operated at a fixed microwave frequency $f_{c}$. This operation mode allows one to trace the radial evolution of the cutoff-layer in plasma discharges with varying density. A typical result is shown in Fig. 6 (program id 160303.013 with $f_{c}=28 \mathrm{GHz}$, cf. also Sec. II). The time traces of $P_{E C R H}$ and $\int n d l$ are shown again in Fig. 6a and b, respectively. The measured amplitude of antenna B is shown in Fig. 6c. At $t_{c}=143 \mathrm{~ms}$ the density increases above the cut-off density $n_{c}=9.7 \cdot 10^{18} \mathrm{~m}^{-3}$ and the amplitude jumps by approximately $5 \mathrm{~dB}$. For $t<t_{c}$ the microwave system is in an interferometric mode and reflections from the wall contribute significantly to the recorded 
signal. The transition from the interferometer to the reflectometer operation is well seen in the CCF amplitudes plotted in Fig. 6d. At $t=t_{c}$ the CCF amplitudes of the displayed antenna combinations make a jump from 0.2 to 0.6-0.7. After the ECRH-switch off at $t=1000 \mathrm{~ms}$, low frequency oscillations with $f \approx 35 \mathrm{~Hz}$ are observed in the radiometer signal, whose origin needs further investigation. The time lags $\tau$ are shown in Fig. 6e and are significant for $t>t_{c}$ only. Since the distance between the reflection point of the antenna combination differs, $\tau$ varies between $2-7 \mu$ s. The radial cut-off position $r_{c} / a$ as calculated from the profile data is shown in Fig. 6f. Since the temporal resolution of the used Thomson scattering system is only $100 \mathrm{~ms}$, the fitted profile data has been interpolated in between the measurement times. The time trace of $r_{c} / a$ has data only if the profile data shows a cut-off at the given frequency $f_{c}$. The profile data suggests that the transition to the reflectometer mode happens at $t \approx 120 \mathrm{~ms}$, which differs significantly from $t_{c}$. Due to the limited radial and temporal resolution of the Thomson scattering system, the reason for this mismatch is hard to investigate. The cut-off layer is mainly centered at $r_{c} / a=0.83$ but
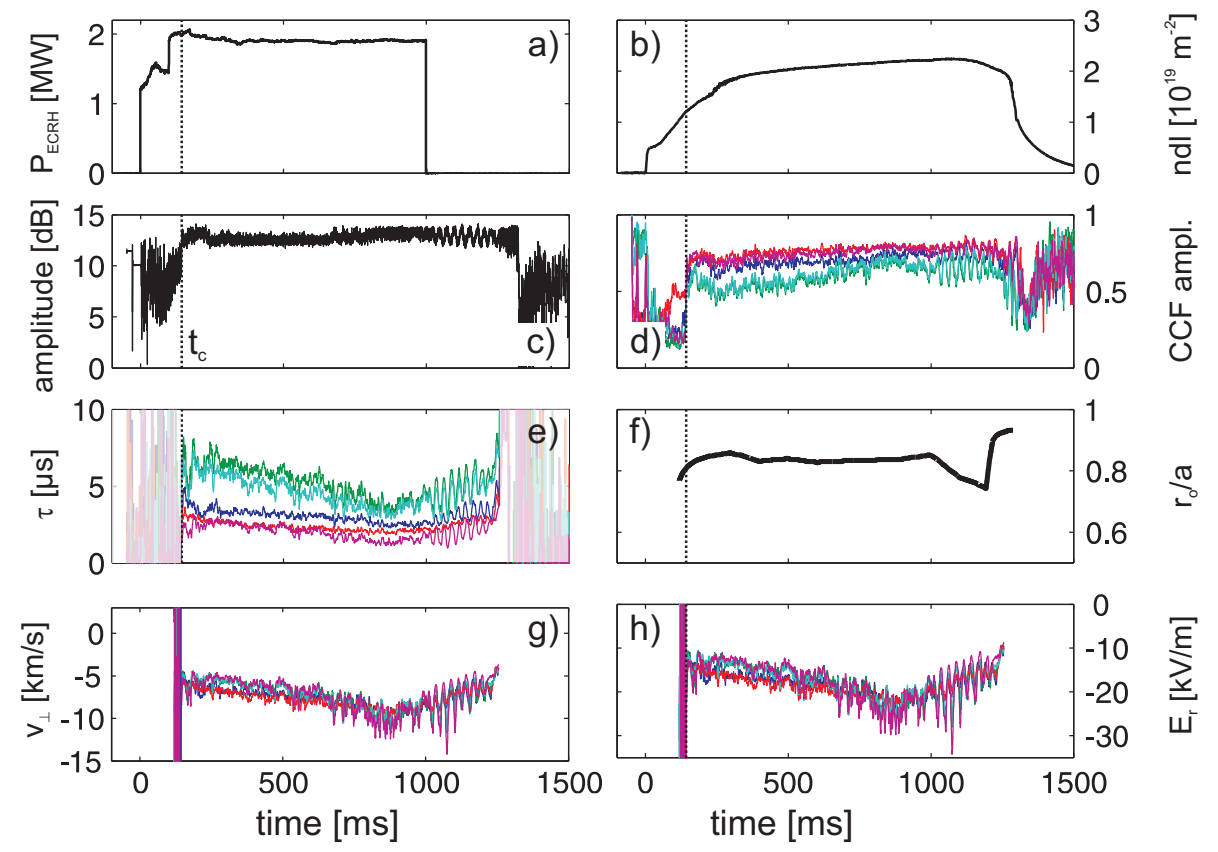

FIG. 6: PCR results for program id 160303.013 with constant $f_{c}=28 \mathrm{GHz}$ : (a) ECRH-power, (b) line-integrated density $\int n d l$, (c) signal amplitude of antenna B, (d) CCF amplitudes, (e) time lags $\tau$, (f) cut-off layer position, (g) perpendicular velocity and (h) local radial electric field $E_{r}$. The dashed vertical line indicates the transition from interferometer to reflectometer operation mode. 
variations are observed for $t<200 \mathrm{~ms}$ and $t>1000 \mathrm{~ms}$. The radial shift of $r_{c} / a$ during plasma build up $(t<200 \mathrm{~ms})$ is small which indicates no drastic changes in the density profile in the plasma edge. After ECRH-switch off at $t=1000 \mathrm{~s}$ the density keeps rising. The typical energy confinement time is $\tau_{E}=130-150 \mathrm{~ms}$ [16]. The perpendicular velocity $v_{\perp}=d_{\perp} / \tau$ is shown Fig. 6g. $v_{\perp}$ is in the electron diamagnetic drift direction and varies in the time interval $t=150-1000 \mathrm{~ms} v_{\perp}$ between -5 and $-10 \mathrm{~km} / \mathrm{s}$. The difference between the $v_{\perp}$ 's obtained for different antenna combinations is small $\left(\Delta v_{\perp} \approx 1.5 \mathrm{~km} / \mathrm{s}\right)$, which gives confidence in the TRAVIS mapping of the reflection points. The estimated perpendicular velocity $v_{\perp}=E_{r} / B+v_{p h}$ is usually a combination of $\boldsymbol{E} \times \boldsymbol{B}$-drift and the phase velocity of the observed fluctuations. As described in Sec. II D the measured propagation of broadband fluctuations in the frequency range $f<100 \mathrm{kHz}$ does not depend on the frequency, which clearly indicates that the dispersive contributions are negligible, such that $v_{\perp} \approx E_{r} / B$ can be assumed. The resulting local $E_{r}$, corresponding to the frequency $f_{c}$, is shown in Fig. $6 \mathrm{~h}$. As the line-integrated density increases, the magnitude of $E_{r}$ increases from $-15 \mathrm{kV} / \mathrm{m}$ at $t=300 \mathrm{~ms}$ to $-20 \mathrm{kV} / \mathrm{m}$ at $t=800 \mathrm{~ms}$. Note that the variations of electron density and temperature profiles (cf. Fig. 3c,d) are mainly in the plasma core.

\section{B. $E_{r}$-profile and comparison with neoclassical predictions}

An access to a broader radial range is achieved by hopping $f_{c}$ in the available microwave band. An example is shown in Fig. 7 (program id 160310.034). At $t=300 \mathrm{~ms}$ the heating
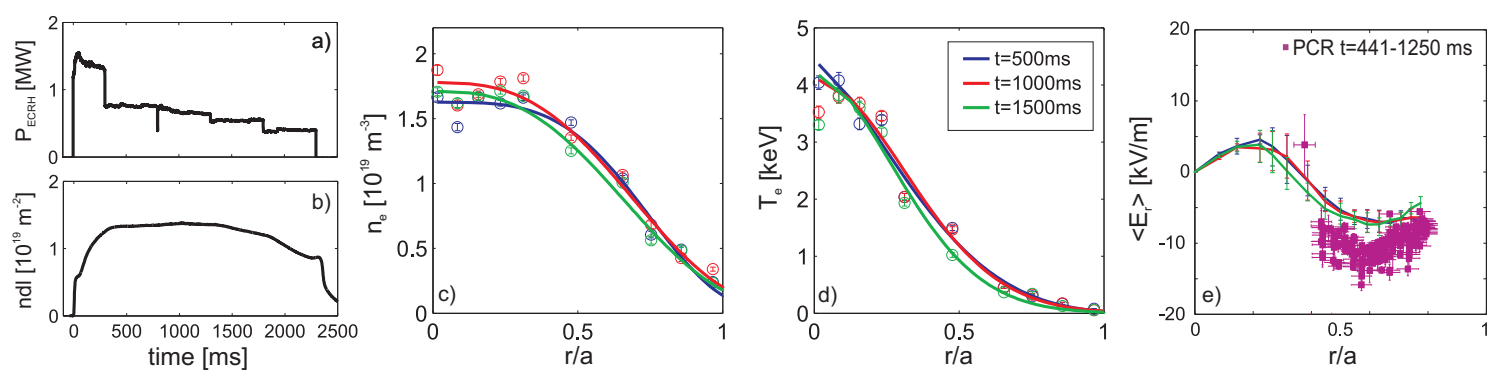

FIG. 7: Parameters and profiles at three time instants of a stepped power discharge (Program id 160310.034): (a) ECRH-power, (b) line-integrated density $\int n d l$, (c) electron density profile $n_{e}$, (d) electron temperature profile $T_{e}$, and (e) radial electric field profile $<E_{r}>$ obtained from PCR (magenta) in the time interval $t=441-1250 \mathrm{~ms}$ and neoclassical estimates. 
power is decreased from $P_{E C R H} \approx 1.4 \mathrm{MW}$ to $0.8 \mathrm{MW}$ (Fig. 7a). For $t=400-1500 \mathrm{~ms}$ the line-integrated density remains almost constant at $\int n d l \approx 1.35 \cdot 10^{19} \mathrm{~m}^{-2}$ (Fig. 7b). Electron density and temperature profiles are shown in Fig. 7c and d, respectively, for three time instants during the flat-top phase. As expected from the $\int n d l$ time trace, the variation of the density profiles is only minor. Due to shape and magnitude of the density profile, the frequency range is limited to $f_{c} \leq 36 \mathrm{GHz}$ which corresponds to $n=0.7-1.6 \cdot 10^{19} \mathrm{~m}^{-3}$ respectively $r / a \approx 0.35-0.75$. Different from the analysis of the previous section, $v_{\perp}$ is not calculated individually for each antenna combination but a joint $v_{\perp}$ is obtained from linear regression of the various $\tau$ and $d_{\perp}$ values. The scattering of $\tau$ in the interval $d t=$ $2 \mathrm{~ms}$ is considered as statistical error. In order to discriminate uncorrelated data, a CCF amplitude threshold of 0.3 is used. The profile of the average radial electric field defined as $\left\langle E_{r}\right\rangle=E_{r} /|\nabla r|$ is displayed in Fig. 7e for the interval $t=441-1250 \mathrm{~ms}$. For $r / a>0.4$, $<E_{r}>$ is negative corresponding to a propagation in electron diamagnetic drift direction. For $r / a<0.4$ a positive $\left\langle E_{r}>\right.$ is observed but the error bars increase. In-between the transition from negative to positive $\left\langle E_{r}>\right.$ the corresponding $\boldsymbol{E} \times \boldsymbol{B}$-velocity is small. The lack of useful data in this region is caused by small correlation amplitudes, which suggests that fluctuations cannot propagate within their auto-correlation time across the antenna reflection points. This is more the case for antenna combinations with larger poloidal distances and the scatter in $v_{\perp}$ increases leading to larger error bars.

For stellarators far from quasi-symmetry [23], the radial electric field is set by ambipolarity of the neoclassical fluxes. In figure Fig. $7 \mathrm{e}<E_{r}>$ obtained from PCR analysis is compared to neoclassical predictions in the monoenergetic limit using the DKES code [7] complemented with momentum-correction techniques [8]. Three different time instants were calculated. A convolution of the monoenergetic transport coefficients is made allowing for $20 \%$ uncertainty in the input profiles of density and temperature as discussed in Ref. [22]. The simulations predict a positive radial electric field in the core region (electron root), as expected for plasmas with low electron collisionality [13]. This result is in agreement with other available measurements [20, 24]. Closer to the plasma edge, the radial region that is well covered by the PCR system, the radial electric field is negative. Here the electron collisionality is much larger than in the core and ion root conditions prevail [25].

The neoclassical calculations and the PCR measurements are in good qualitative agreement. The measured $<E_{r}>$ corresponds to the electron- and ion-root regime with a transi- 

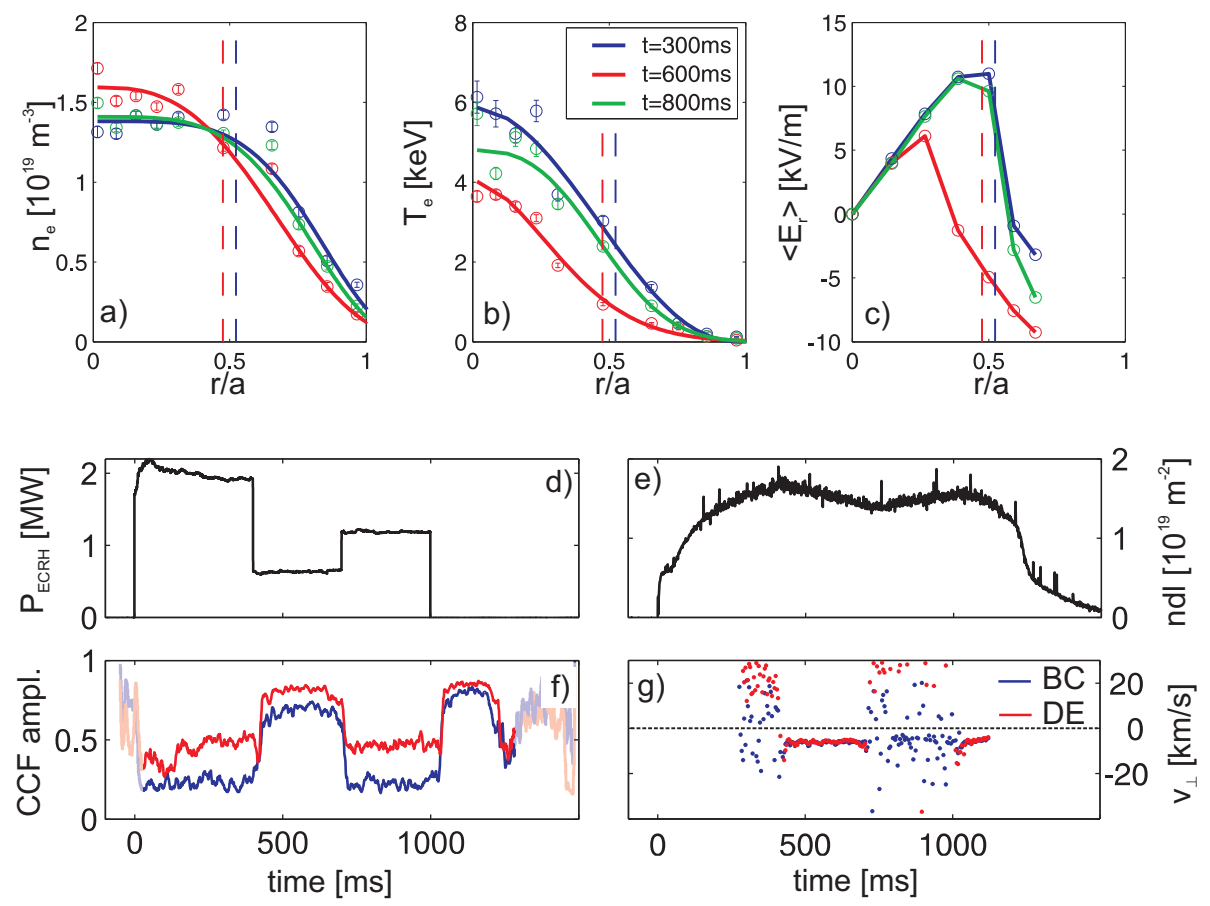

FIG. 8: (a) Radial profiles of $n_{e}$, (b) $T_{e}$ and (c) predicted $<E_{r}>$ for three time instants $300 \mathrm{~ms}$ (blue lines), $600 \mathrm{~ms}$ (red lines) and $800 \mathrm{~ms}$ (green lines). (d) Time trace of $P_{E C R H}$, (e) $\int n d l$, (f) PCR CCF amplitude, and (g) PCR $v_{\perp}$ for antenna combinations BC (blue lines) and DE (red lines). The dashed lines in (a-c) indicate the measurement location at $t=300 \mathrm{~ms}$ and $t=600 \mathrm{~ms}$ (Program id 160309.024).

tion region that matches quite well the localization predicted by the neoclassical calculations. The slope of the decrease of $\left\langle E_{r}>\right.$ at $r / a \sim 0.6-0.7$ (ion-root regime) does also agree. The magnitude of $\left\langle E_{r}\right\rangle$ measured with $\mathrm{PCR}$ is however approximately a factor of 1.5 larger when compared to the neoclassical prediction. This deviation is in the plasma edge, where $T_{e} \approx T_{i}$ might be caused by by neoclassical corrections to the monoenergetic approximation $[9,11,12]$ by the presence of impurities (which increase the species collisionality and also may contribute to the ambipolarity equation) or by a small turbulent contribution that may become non-negligible for outer radial positions. The PCR diagnostic in the transition region where $\left\langle E_{r}>\right.$ flips sign is challenging owing to the small propagation velocity of the fluctuations.

This issue can be addressed in more detail in discharges with fixed frequency $f_{c}$ but varying heating power. The aim is to study the modification of the radial ambipolar electric field for different change in electron and ion transport caused by the varying collisionality. 
Fig. 8 summarizes the main PCR findings (program id 160309.024). The heating power $P_{E C R H}$ is changed in steps from $P_{E C R H}=2 \mathrm{MW}$ for $t<400 \mathrm{~ms}$ to $P_{E C R H}=0.6 \mathrm{MW}$ for $400<$ $t<700 \mathrm{~ms}$ to $P_{E C R H}=1.2 \mathrm{MW}$ for $700<t<1000 \mathrm{~ms}$ (cf. Fig. 8d). During the heating power changes the line-integrated density shows only minor modifications (cf. Fig. 8e). The radial profiles of $n_{e}, T_{e}$ and predicted $\left\langle E_{r}>\right.$ are shown in Fig. 8a-c for three time instants $t=$ $[300,600,800] \mathrm{ms}$ that correspond to the three different heating powers . While the density profile remains almost constant, the central $T_{e}$ decreases from $6 \mathrm{keV}$ at $t=300 \mathrm{~ms}$ to $4 \mathrm{keV}$ at $t=600 \mathrm{~ms}$ and recovers to $5 \mathrm{keV}$ at $t=800 \mathrm{~ms}$. The change in the $T_{e}$-profile has a strong response in the predicted $\left\langle E_{r}>\right.$-profiles (Fig. 8c). For all time instants the electron-root regime in the plasma core and and the ion-root regime at larger radii prevail. But in the high power heating phase the electron-root extends to $r / a \approx 0.6$ while in the low power heating phase its radial extent decreases to $r / a \approx 0.35$. The PCR frequency during these measurements is kept fixed at $f_{c}=32 \mathrm{GHz}$, corresponding to $n_{c} \approx 1.25 \cdot 10^{19} \mathrm{~m}^{-3}$. The location of the cut-off layer is indicated in Fig. 8a-c as dashed lines for $t=300 \mathrm{~ms}$ and $t=600 \mathrm{~ms}$. Despite the shallow density profile the cut-off layer is still sufficiently radially localized as two-dimensional full wave simulations using the IPF-FD3D code [26] reveal (not shown here). From the FWHM of the squared wave electric field at the cut-off a resolution of $\Delta r \approx 18 \mathrm{~mm}$ is obtained. The cut-off position decreases slightly from $r_{c} / a=0.52$ to $r_{c} / a=0.48$ in the low and high heating power phase, respectively. In Fig. 8c it is seen that the cut-off layer is located in the transition region between the electron- and ion-root for $t<400 \mathrm{~ms}$ (note that this region can be located only approximately by radially local neoclassical simulations), while it is only in the ion-root regime for $400<t<700 \mathrm{~ms}$. The time trace of the CCF amplitude for the two antenna combinations BC and DE is shown in Fig. 8f. The high correlation amplitude at $t<0$ and $t>1200 \mathrm{~ms}$ (shaded region in Fig. 8f) is caused by instrumental artefacts (cf. Fig. 6e). A cut-off in the plasma does only exist in the time interval $t \approx 250-1200 \mathrm{~ms}$ as the calculated $v_{\perp}$ in Fig. $8 \mathrm{~g}$ suggests. The poloidal distance of the reflection points for BD is a factor of 2 larger than for $D E$, e.g. $d z_{B D}=3.2 \mathrm{~cm}$ and $d z_{D E}=1.6 \mathrm{~cm}$ at $t=300 \mathrm{~ms}$. In the high power heating phase the CCF amplitude of BC is close to the noise limit while for DE a significant CCF amplitude of about 0.5 is observed. In the low power heating phase both antenna combinations have much higher CCF amplitudes (typically larger than 0.7 ). In the low power heating phase the resulting perp. velocities have positive values and are in good agreement for both antenna combinations (cf. Fig. 8f). This corresponds to the ion-root 
regime. In the high power heating phases $v_{\perp}<0$ for antenna combination DE, which clearly indicates the electron-root regime.

\section{High-frequency fluctuations}

Until this point it has been is assumed that the velocity measured with the PCR system is predominantly given by the $\boldsymbol{E} \times \boldsymbol{B}$-velocity and that the contribution of the phase velocity of the fluctuations is negligible. This assumption is supported by the results presented in Figs. 5b-c and 6 in which broadband fluctuations in the frequency range $f<100 \mathrm{kHz}$ cause a linear slope of the cross-phase. Reconsidering Fig. 7, it is evident that in the transition between electron- and ion-root region this assumption does not hold. For a deeper investigation the cut-off layer position $r_{c} / a$ and the coherence of antenna combination DE are shown in Fig. 9 for one hopping sweep $f_{c}=22-36 \mathrm{Ghz}$ in the time interval $t=821-1120 \mathrm{~ms}$. The position of the transition from electron- and ion-root regime, as obtained from the PCR analysis, is indicated in Fig. 9a as red line $\left(r_{c} / a=0.4\right)$. The coherence in the frequency range $\pm 250 \mathrm{kHz}$ is shown in Fig. 9b. While for $r_{c} / a>0.4$ the coherence is distributed over a broad band in the range $f<100 \mathrm{kHz}$, for $r_{c} / a<0.4$ mainly high-frequency fluctuations with $f>150 \mathrm{kHz}$ contribute to the coherence. A better understanding of the high-frequency fluctuations is essential to interpret the positive $\left\langle E_{r}>\right.$ discussed in the previous section.

For a further analysis data of discharges with higher constant heating power $P_{E C R H}=$

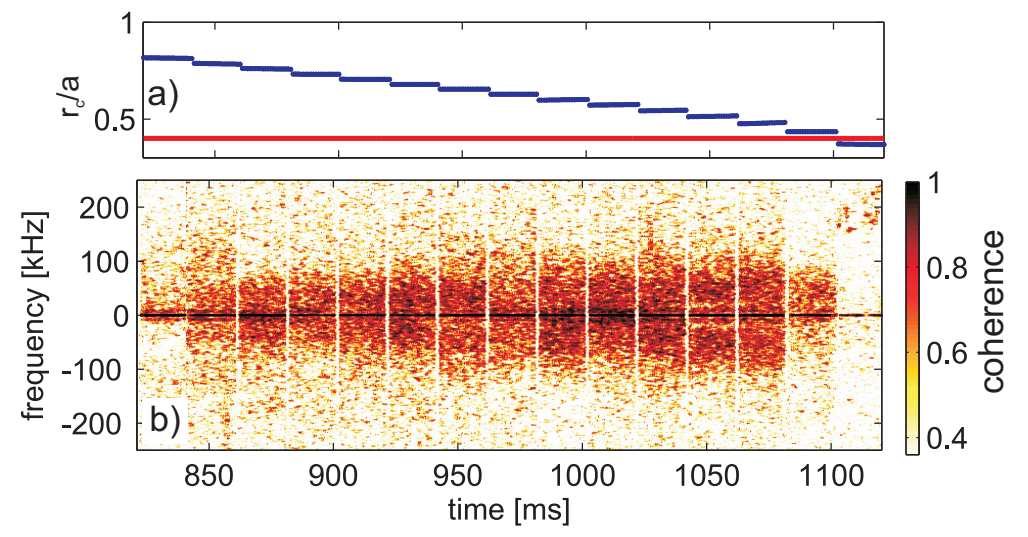

FIG. 9: (a) Radial cut-off layer position $r_{c} / a$ (a) and (b) coherence of antenna combination DE for one hopping sweep (program id 160310.034). The red line in a) indicates the transition from the electron- to ion-root as measured with the PCR system (cf. Fig. 7e). 

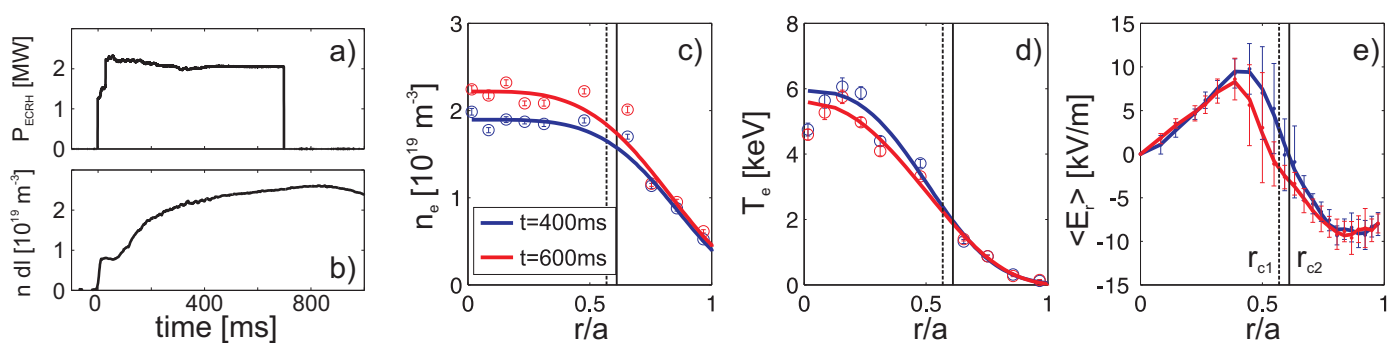

FIG. 10: (a) Time traces of $P_{E C R H}$ and (b) $\int n d l$ and radial profiles of $n_{e}$ (c), $T_{e}$ (d) and predicted $\left\langle E_{r}>\right.$ (e) for two time instants $t=[400,600] \mathrm{ms}$. The lines in (c-e) indicate the measurement locations $r_{c 1}$ and $r_{c 2}$ addressed in the text (program id 160309.013).

$2 \mathrm{MW}$ are considered. The time traces of $P_{E C R H}$ and $\int n d l$, and radial profiles of $n_{e}, T_{e}$ and $\left\langle E_{r}>\right.$ for two time instants $t=[400,600] \mathrm{ms}$ are compiled in Fig. 10. In the analysis we concentrate on two consecutive hopping frequencies: $f_{c 1}=36 \mathrm{GHz}$ and $f_{c 2}=37 \mathrm{GHz}$ in the time intervals $t_{c 1}=421-431 \mathrm{~ms}$ and $t_{c 2}=431-441 \mathrm{~ms}$, respectively. The positions of the cut-off layers $r_{c 1} / a=0.61$ and $r_{c 2} / a=0.57$ are close to the transition region, as indicated in Fig. 10e by vertical lines. The coherence and cross-phase of antenna combination DE is shown in Fig. 11a-b for $f_{c 1}$ and $f_{c 2}$. For $f_{c 1}$ the peak coherence is larger than 0.7 and symmetrically centered around $f=0$. The corresponding cross-phase has a positive slope which indicates propagation in the electron diamagnetic drift direction (ion-root regime). For $f_{c 2}$ the central peak coherence decreases to 0.4 and an additional peak at $f \approx 180 \mathrm{kHz}$ with a coherence of 0.36 is observed. The cross-phase for $f_{c 2}$ clearly indicates that the lowfrequency components $|f|<50 \mathrm{kHz}$ have the same propagation as for $f_{c 1}$. The high frequency component, however, has a negative slope which corresponds to propagation in the iondiamagnetic direction associated with a positive $\left\langle E_{r}\right\rangle$. The electromagnetic character of the high-frequency fluctuations becomes evident if Mirnov data is considered. Four Mirnov coils have been operated that are located in the triangular plane $\left(\varphi=177^{\circ}\right)$ with poloidal positions ranging from $\theta=80-132^{\circ}$ [21]. A coherence spectrogram of two adjacent Mirnov coils is shown in Fig. 11c. The coherence is dominated by low frequency fluctuations at $f \approx 7 \mathrm{kHz}$ which are addressed in the next section. In addition high-frequency bands are observed most dominantly in range $f=150-200 \mathrm{kHz}$. As the line-integrated density increases (cf. Fig. 10a) the frequency decreases. This scaling behavior and the frequency range itself are indicating that these electromagnetic fluctuations are caused by Alfvén waves 

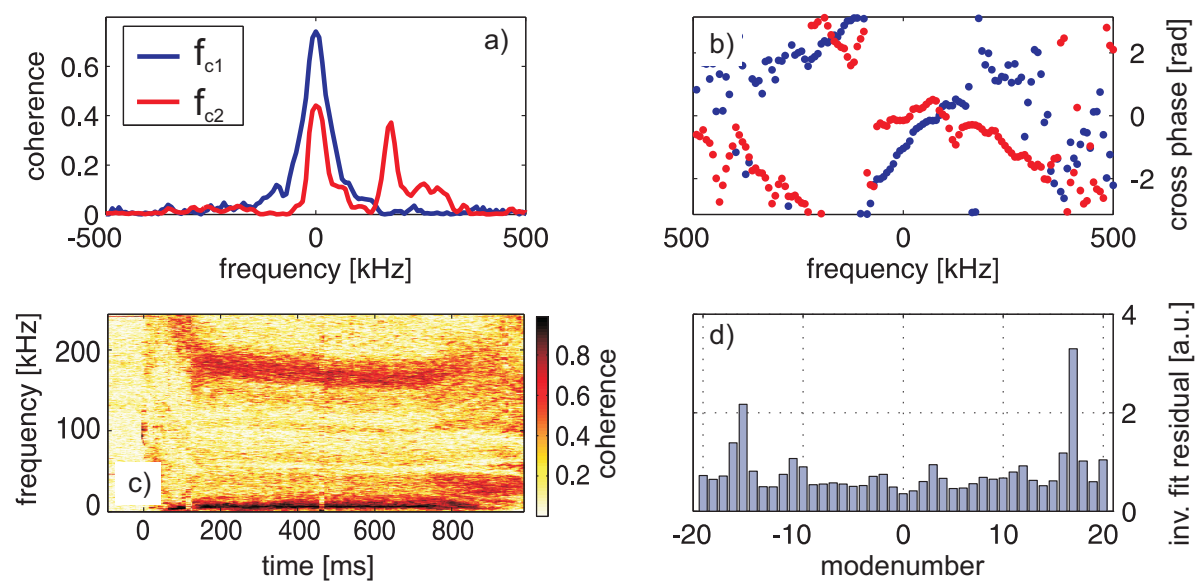

FIG. 11: (a) PCR coherence and (b) cross-phase of antenna combination DE for $f_{c 1}$ and $f_{c 2}$. Coherence spectrogram obtained from Mirnov coils (c) and inverse modenumber fit residual (d) (program id 160309.013).

(Alfvén velocity $v_{a} \sim 1 / \sqrt{n}$ ). The radial location of the measured magnetic field fluctuations is a priori unknown and a reconstruction with only four coils is impossible. However, the radial localization can be determined by PCR measurements. For the discussed cut-off layer positions $r_{c 1,2} / a \approx 0.6$ the PCR and the Mirnov coils are connected in $\theta$ with a connection length of $L=11 \mathrm{~m}$ assuming the measured magnetic field fluctuations originate from the position on the flux surface with the smallest distance to the actual Mirnov coil position. The cross-correlation analysis between the PCR and Mirnov data in the interval $t_{c 2}$ results in a CCF amplitude of 0.25 which exceeds the noise level but is governed by the low-frequency fluctuations. Using the latter assumption, the Mirnov data can be used to obtain a poloidal modenumber of the high-frequency fluctuations. For this purpose the Mirnov data is filtered around $f_{w}=180 \mathrm{kHz}$ using wavelet bases and the phase evolution is fitted assuming harmonic perturbations $\tilde{B}=\cos \left(m\left(\theta-\omega_{w} t\right)\right)$ with $|m| \leq 20$. The inverse fit residual (Fig. 11d) for the various modenumbers suggests that the high-frequency component is caused by fluctuations with poloidal modenumber $m=+16$.

To compare measured frequencies with theoretical predictions, the continuous Alfvén spectrum has been calculated for this discharge using the CONTI code [27]. To account for the geodesic acoustic mode (GAM) upshift of the frequencies, the slow sound approximation has been used [27]. Close to the plasma center, where $\beta$ is relatively high, the upshift is relevant in the low frequency range but negligible for frequencies close to the elliptical 
Alfvén Eigenmodes (EAE) gap of considered here. The continuous Alfvén spectrum is shown in Fig. 12a. For the experimentally observed poloidal mode number $m=16$, several global EAE can be found, having different numbers of radial knots and different degrees of continuum interaction. An example EAE at $f=201 \mathrm{kHz}$ with one radial knot is shown in Fig. 12b for example. Fig. 12c shows an EAE mode with $f=197 \mathrm{kHz}$ without radial knots but exhibiting strong interaction with the continuum branches of $m=20 / m=22, n=-12$. It is an interesting question if the experimental observation indeed supports the excitation of modes from the stable Alfvén continuum. Calculating the power transfer between an EAE mode and electrons with CKA-EUTERPE [29], it could be shown that an excitation of the mode by fast electrons is in principle possible. The mode could be driven unstable by the considerable electron temperature gradient via a resonant wave-particle interaction. As the Alfvén speed is about a factor of three smaller than that of the electrons, a resonance with circulating particles can be almost safely excluded. More likely seems to be the a resonance of the mode with the bounce or drift motion of electrons from a certain portion of phase space. More experimental evidence is needed to rule out speculation and allow a thorough investigation of the phenomenon. phenomenon.

\section{Low-frequency fluctuations}

The coherence analysis of the Mirnov data (Fig. 11c) already revealed low-frequency components in the fluctuation spectrum. The observed frequency is centered around $f \approx$ $5-10 \mathrm{kHz}$. The associated density fluctuations are also observed in the PCR measurements as the following analysis supports (program id 160303.013 already addressed in in Sec. III A). Due to the fixed frequency operation at $f_{c}=28 \mathrm{kHz}$ the cut-off layer position is
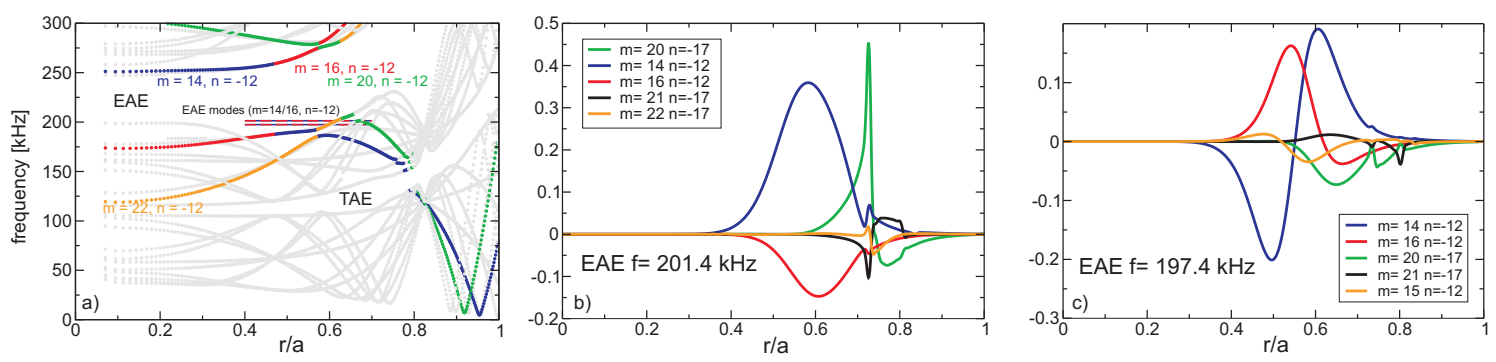

FIG. 12: (a) Calculated continuous Alfvén spectrum, (b) EAE at $f=201 \mathrm{kHz}$, (c) EAE at $f=$ $197 \mathrm{kHz}$. 

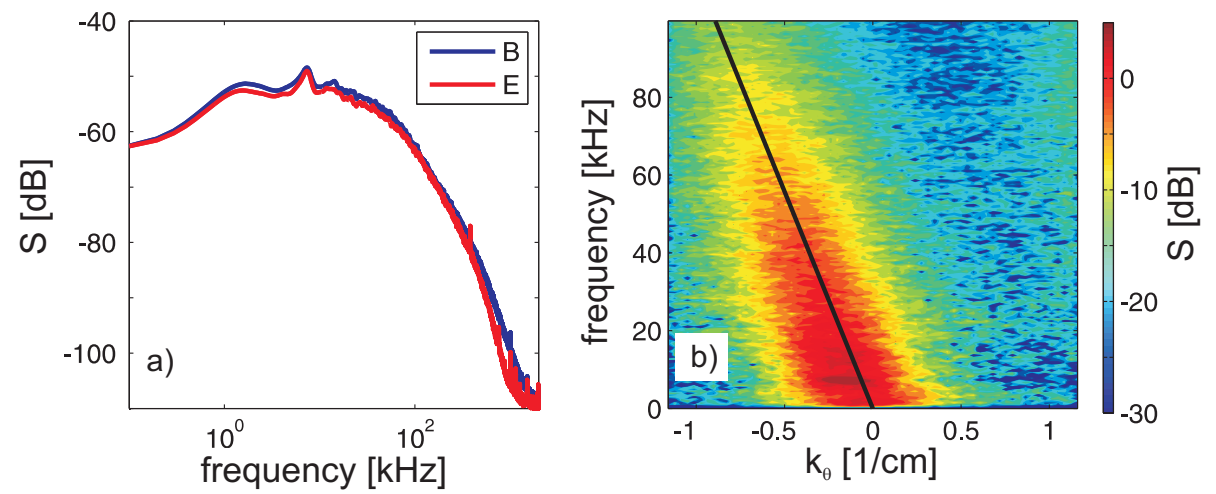

FIG. 13: (a) Fluctuation spectra and (b) frequency-wavenumber spectrum $S\left(k_{\theta}, f\right)$ (b) obtained from PCR using antenna combination BE. The black line in (b) indicates the $\boldsymbol{E} \times \boldsymbol{B}$-velocity (program id 160303.013).

approximately constant at $r_{c} / a=0.83$ in the considered time interval $t=400-600 \mathrm{~ms}$. The fluctuation spectra of antenna combination BE are shown in Fig. 13a. The spectra are characterized by a power-law decay for $f>100 \mathrm{kHz}$ and a low-frequency peak at $f \approx 7 \mathrm{kHz}$. From the two separate measurements, a frequency-wavenumber spectrum $S\left(k_{\theta}, f\right)$ can be computed [10]. The poloidal separation of the reflection points is $2.7 \mathrm{~cm}$. The result is illustrated in Fig. 13b. $S\left(k_{\theta}, f\right)$ is dominated by broadband fluctuations that propagate with $\boldsymbol{E} \times \boldsymbol{B}$-velocity in the electron diamagnetic direction (ion root). The poloidal component of the $\boldsymbol{E} \times \boldsymbol{B}$-velocity, estimated from the PCR results (Fig. 6h), is shown as black line. The low-frequency fluctuations that are observed at $f \approx 7 \mathrm{kHz}$ have a poloidal wavenumber $k_{\theta}=0.1-0.2 \mathrm{~cm}^{-1}$ which corresponds to modenumbers $m=k_{\theta} r_{c}=4-8$. A similar analysis of the Mirnov data (presented in the last section) suggests a modenumber of $m=5$ which agrees with the PCR findings. Other experimental programs during which $f_{c}$ is changed indicate that the low-frequency fluctuations are observed in a radial range $r / a \approx 0.7-0.9$.

The origin of the low-frequency fluctuations is investigated in the limit of ideal MHD using the CAS3D code [4]. The equilibrium data has been obtained from free-boundary VMEC calculations with five field periods maintaining stellarator symmetry. Equilibrium properties breaking the five-fold symmetry are ruled out in the simulations. For the experimental pressure profiles (Fig. 10, $t=400 \mathrm{~ms}$ ) an average $\langle\beta>=0.284 \%$ is obtained from VMEC. The rotational transform profile results from a calculation with vanishing toroidal current. It is shown in Fig. 14a. In the CAS3D computation 71 Fourier harmonics for the scalar 

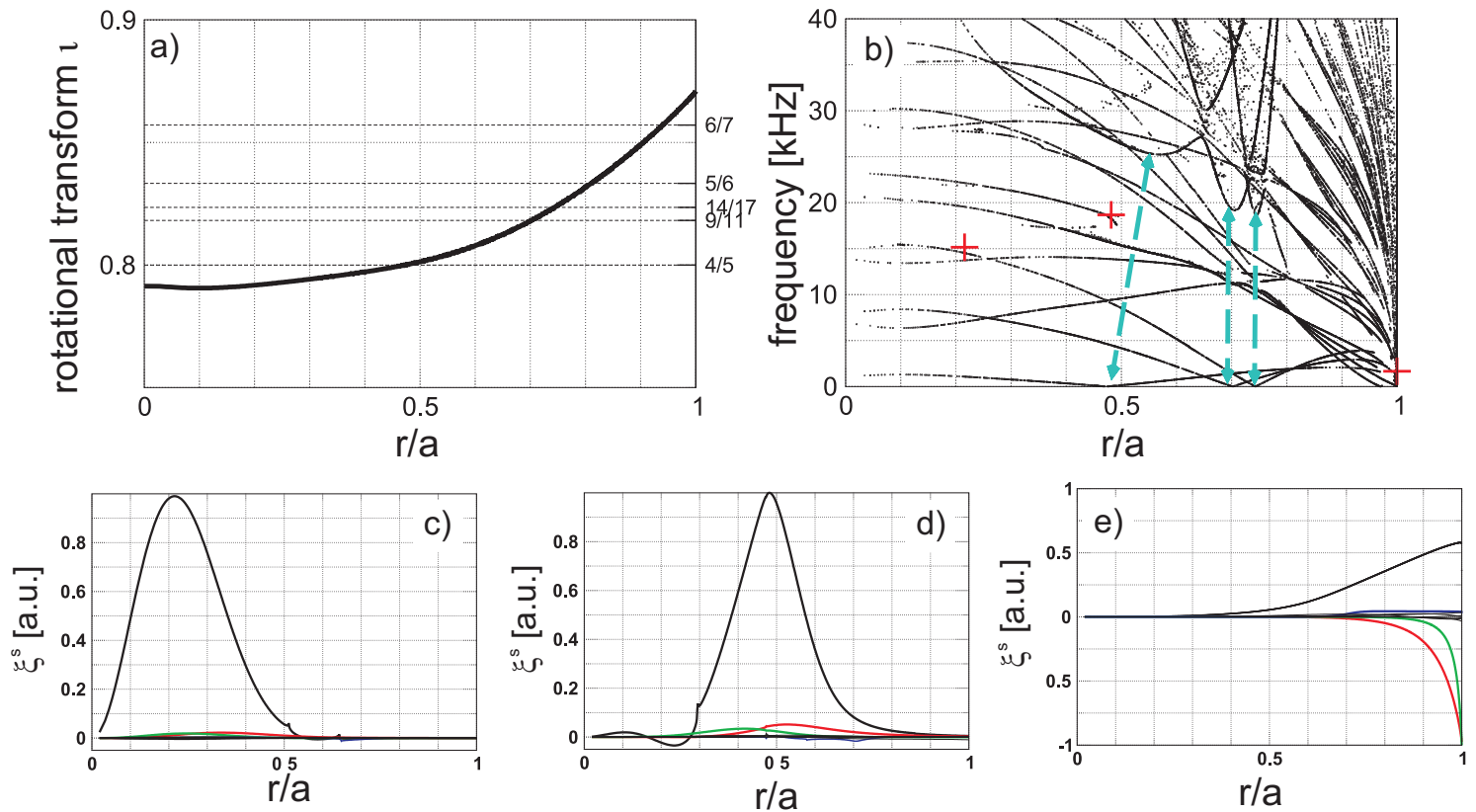

FIG. 14: Ideal MHD computation using the CAS3D code (program id 160309.013): (a) VMEC rotational transform, (b) stable spectrum and normal displacement $\xi^{s}$ of the 10 strongest harmonics at (c) $f \approx 15 \mathrm{kHz},(\mathrm{d}) f \approx 18 \mathrm{kHz}$, and (e) $f \approx 1.6 \mathrm{kHz}$. The $n=4, m=5$ perturbation is shown as thick black line.

normal displacement $\xi^{s}$ and 1280 equidistant flux intervals for the radial discretization are used. The stable spectrum in the range $f<40 \mathrm{kHz}$ is shown in Fig. 14b. Sound continuum branches fill this frequency range (black dotted lines). By way of example, the $\beta$-induced gaps are indicated for three resonant rational rotational transforms, $\iota=4 / 5,9 / 11$, and 14/17 (cyan arrows in Fig. 14b). They originate from the crossing of sound and Alfvén continuum branches, eliminated by the 'avoid-crossing phenomenon' in non-cylindrical configurations [31]. In the present case, the $\beta$-induced gap is about 20 to $30 \mathrm{kHz}$ wide in the outer part of the plasma, $r / a \leq 0.5$. Stable, global, radially extended perturbations are present in this frequency range. Three selected, essentially $n=4, m=5$ perturbations are shown in Fig. 14c$\mathrm{e}$ in form of the dominant Fourier harmonics of the respective normal displacement, i.e. the ideal MHD displacement normal to flux surfaces. Their frequencies are indicated in Fig. $14 \mathrm{~b}$ as red crosses. At very low frequency $f \approx 1.6 \mathrm{kHz}$ a perturbation exists with the maximum amplitudes of the normal displacement Fourier harmonics at the plasma edge. In this perturbation, the $n=14, m=16$ and $n=9, m=10$ harmonics also contribute. The perturbations at $f \approx 15 \mathrm{kHz}$ and $f \approx 18 \mathrm{kHz}$ are essentially $n=4, m=5$ with very small side- 
bands. At the plasma boundary, the $n=4, m=5$ amplitude is very small but non-zero. Despite their small amplitudes near the edge, these perturbations can be excited from the plasma edge.

In terms of localization, the CAS3D results for the $n / m=4 / 5$ perturbation at $f \approx 18 \mathrm{kHz}$ agree fairly well with the experimental PCR findings. The difference in the frequencies is most likely attributed to the Doppler-shift: if the Doppler-shift is taken into account both frequencies agree quite well if the stable mode propagates poloidally in the ion diamagnetic drift direction (cf. Fig. 13b).

\section{SUMMARY AND CONCLUSION}

It has been demonstrated that the PCR system is a versatile diagnostic to investigate the radial electric field and density fluctuation characteristics. Due to the low plasma density in the first operation phase of W7-X, a large fraction of the profile has been probed. The presented measurements of the PCR systems clearly revealed the electron- and ion-root regime in the plasma core and edge, respectively. In the ion-root regime, $\left\langle E_{r}>\right.$ obtained from PCR is in fair agreement with neoclassical predictions. In the electron-root regime if has been found that electromagnetic high-frequency fluctuations can strongly influence the observed coherence. In general the PCR analysis is challenging in the transition region from the electron- to ion-root since the $\boldsymbol{E} \times \boldsymbol{B}$-velocity is too small for the fluctuations to propagate within their correlation time across the antennae reflection points. In the electron root regime, with large $E_{r}>0$, the density profile is shallow which strongly reduces the radial resolution of the PCR system. As a consequence, the PCR measurements are mainly restricted to the ion-root regime. The analysis of PCR and Mirnov data in comparison with CONTI simulations suggest that the high-frequency fluctuations at $f \approx 200 \mathrm{kHz}$ are related to Alfvén-waves with poloidal modenumber $m=16$. The excitation of Alfvén-waves by fast electrons is in principle possible but needs further investigation. The low-frequency fluctuation spectrum is dominated by coherent electromagnetic $m=5$ fluctuations with $f \approx$ $5-10 \mathrm{kHz}$ observed at $r / a \approx 0.7-0.9$. A CAS3D simulation suggests that these fluctuations can be attributed to global stable $n / m=4 / 5$ perturbations. The calculations have been performed using the ideal CAD coil geometries. Considering the as-built coil geometry and the deformation due to electromagnetic forces the $\iota$-profile is typically $2 \%$ smaller [30] which 
shifts the 4/5-resonance from $r / a=0.5$ to $r / a=0.7$ in better agreement with the experimental observation. However, since the $n / m=4 / 5$ perturbation does not obey $N=5$ stellarator symmetry it has not been considered so far in the equilibrium calculations.

\section{Acknowledgement}

The authors thank JA Alonso and K Rahbarnia for providing the Mirnov data and fruitful discussions.

This work has been carried out within the framework of the EUROfusion Consortium and has received funding from the Euratom research and training programme 2014-2018 under grant agreement No 633053. The views and opinions expressed herein do not necessarily reflect those of the European Commission.

[1] H Wobig Plasma Phys. Control. Fusion 35903 (1993).

[2] CD Beidler et al. Nucl. Fusion 51076001 (2011).

[3] G Grieger et al. Phys. Fluids B 42081 (1992).

[4] C Schwab Phys. Fluids B5 3195 (1993).

[5] P Helander et al. Plasma Phys. Control. Fusion 54124009 (2012).

[6] JH Proll et al. Phys. Plasmas 20122506 (2013).

[7] SP Hirshman et al. Phys. Fluids 292951 (1986).

[8] H Maaßberg et al. Phys. Plasmas 16072504 (2009).

[9] I Calvo et al. Phys. Plasmas submitted (2016).

[10] CP Ritz et al. Rev. Sci. Instrum.591739 (1988).

[11] S Satake et al. Plasma and Fusion Research 1002 (2006).

[12] M Landreman et al. Phys. Plasmas 21042503 (2014).

[13] M Yokoyama et al. Nucl. Fusion 471213 (2007).

[14] TS Pedersen et al. Nucl. Fusion 55126001 (2015).

[15] A Krämer-Flecken et al. Rev. Sci. Instrum. 81113502 (2010).

[16] M Hirsch et al. IAEA conference 2016 Kyoto, Japan, submitted to Nucl. Fusion.

[17] E Paschet al. Rev. Sci. Instrum. 87101063 (2016). 
[18] NB Marushchenko et al. Computer Physics Communications 185165 (2014).

[19] HJ Hartfuß and T Geist Fusion plasma diagnostics with mm-waves Wiley (2013).

[20] N Pablant et al 43rd EPS Conference on Plasma Physics Vol. 40A, P4.013 (2016).

[21] K Rahbarnia et al 43rd EPS Conference on Plasma Physics Vol. 40A, P4.011 (2016).

[22] JL Velasco and F Castejn Plasma Phys. Control. Fusion 54, 015005 (2012).

[23] I Calvo et al. Plasma Phys. Control. Fusion 55, 125014 (2013).

[24] A Dinklage et al. 43rd EPS Conference on Plasma Physics Vol. 40A, O2.107 (2016).

[25] A Dinklage et al. Nucl. Fusion 53, 063022 (2013).

[26] C Lechte IEEE Trans. Plasma Sci.37, 1099 (2009).

[27] A Könies and D. Eremin Phys. Plasmas 17, 012107 (2010).

[28] A Könies 10th IAEA Technical Meeting on "Energetic Particles in Magnetic Confinement Systems", Kloster Seeon (2007).

[29] T Fehér "Simulation oft he interaction between Alfvén waves and fast particles", $\mathrm{PhD}$ thesis, University of Greifswald (2014).

[30] T Andreeva et al. 29th Symposium on Fusion Technology 2016, Prague, Czech Republic, submitted to Fusion engineering and design.

[31] DA D'Ippolito and JP Goedbloed Plasma Physics 25, 537 (1983).

[32] A Krämer-Flecken et al. Nucl. Fusion 57, 066023 (2017). 